\title{
IMMUNOEPIDEMIOLOGY OF PLASMODIUM VIVAX MEROZOITE SURFACE PROTEIN-4 (PVMSP-4) IN SRI LANKA: A PRELIMINARY STUDY
}

\author{
Upadari Dissanayake ${ }^{1}$, Sajani Dias ${ }^{2}$, Hannah Polson $^{3}$, Shirley Longacre ${ }^{3}$ and \\ Preethi V Udagama-Randeniya ${ }^{2} *$ \\ ${ }^{1}$ Institute of Biochemistry, Molecular Biology and Biotechnology, University of Colombo,Sri Lanka. \\ ${ }^{2}$ Department of Zoology, University of Colombo, Sri Lanka. \\ ${ }^{3}$ Laboratoire de Vaccinologie Parasitaire, Institut Pasteur, Paris, France. \\ Accepted 17 June 2008
}

\begin{abstract}
Natural antibody (IgG + IgM) responses to putative vaccine candidate, Plasmodium vivax Merozoite Surface Protein-4, in acute vivax malaria patients in endemic and non-endemic areas of Sri Lanka, were assayed by indirect ELISA using PvMSP-4 baculovirus derived recombinant protein. The study populations were from two vivax malaria endemic areas, Anuradhapura and Kataragama and from a nonendemic area, Colombo. PvMSP4 appeared to be immunogenic in all test populations with a prevalence of $80 \%$, 62\% and 62\% from Colombo, Anuradhapura and Kataragama, respectively. A significantly higher prevalence and antibody magnitude was recorded for non-endemic Colombo, but with no significant differences between individuals previously not exposed to malaria and those with prior exposure. A positive trend in responders from Kataragama was evident between the antibody prevalence and previous exposure. We previously reported the antibody response to PvMSP-1 (p19 and p42) using the same battery of sera, and when comparisons were drawn, more than $80 \%$ from each test area responded to at least one of the three antigens tested. Importantly, $7 \%, 11 \%$ and $4 \%$ of individuals from Colombo, Anuradhapura, and Kataragama, respectively, preferentially recognized PvMSP-4. Responders to MSP-1p42 and p19 in paired combination showed higher prevalence compared with combinations of MSP-1 with MSP-4.
\end{abstract}

\section{INTRODUCTION}

The global burden of malaria due to Plasmodium vivax is $\sim 70-80$ million cases annually. $P$. vivax accounts for $>50 \%$ of all malaria cases outside Africa, and is endemic in the Middle East, Asia and Western Pacific, with a lower prevalence in Central and South America (Mendis et al., 2001). Coupled with robust transmission of $P$. vivax in temperate regions, evidence suggests that the relative burden of $P$. vivax is expected to increase in the coming decades (Mendis et al., 2001). As malaria transmission rates are low in most areas where $P$. vivax is prevalent, affected human populations achieve little natural immunity to vivax, and all ages are at risk (Mendis et al., 2001).

Malaria has been a major public health problem and a socio-economic burden in Sri Lanka (Konradsen et al., 2000). Estimated populations of 10.5 million live in malaria endemic areas of the island (Wickremasinghe, 2002)). P. vivax infections accounted for $88 \%$ of the cases recorded for 2002 and $P$. falciparum infections comprised the balance (Annual Health Bulletin, 2002). Malaria is endemic in the dry and the intermediate zones of the island (Annual Health Bulletin, 2002). The low transmission of malaria in Sri Lanka is accompanied by a fluctuating incidence of the disease resulting in "unstable" malaria (Rajendram and Jayewickreme, 1951, Mendis et al., 1990). The low level of acquired immunity present a clinical pattern of the disease in the population which contrasts to that found in areas with "stable" transmission (Mendis et al., 2001).

Control of malaria is becoming increasingly difficult due to resistance of parasites to commonly used drugs as well as resistance of vectors to commonly used insecticides (Waters, 2006). Although alternative control measures such as impregnated bed nets show some promise, it is agreed that an effective subunit vaccine would be an important advance in combating this disease

\footnotetext{
*Corresponding author’s email: dappvr@yahoo.com
} 
(Hoffman et al., 1991). Such an effective malaria vaccine containing multiple proteins from all stages of parasite development, including the asexual blood stage, should elicit an immune response that either destroys the parasite or its infected host cell, or inhibit a function that is crucial to its survival (Hoffman et al., 2002). Thus, subunit vaccines composed of multiple antigen constructs may gain greater protection against malaria. Considerable effort is devoted to identify asexual stage antigens that are exposed proteins of the parasite, as these would induce host protective responses and would be promising targets for vaccines. Immune responses to such antigens were shown to interfere with merozoite invasion in vitro and in some cases to offer protection from infection in animal models (Anders et al., 1993).

Erythrocyte invasion is a multi step process involving a series of specific interactions. The Plasmodium merozoite represents the invasive form of the asexual blood stage. Proteins exposed on the merozoite surface have been considered as potential vaccine candidates (Anders and Saul, 2000). Nine $P$. vivax merozoite surface proteins have been identified to date, all being named in accordance with their $P$. falciparum orthologues (Barnwell et al, 1999).

P. falciparum merozoite surface protein 4 (PfMSP-4) is a relatively newly identified protein, with an observed molecular mass of $40 \mathrm{kDa}$ (Marshall et al., 1997). Nucleotide sequencing studies revealed a glycosylphosphatidylinositol (GPI) - anchored integral membrane protein that possesses an epidermal growth factor (EGF)-like domain at the carboxyl terminus of the protein (Marshall et al., 1997). A study done by Wang et al. (1999) provided the first evidence that the antigenicity of PfMSP-4 is conformation dependent and thus correct folding of the EGFlike domain is crucial. MSP-4 is immunogenic in laboratory animals (Wang et al., 1999), and antibodies raised to it can inhibit parasite growth in vitro (Wang et al., 2003).

In $P$. vivax which is phylogenetically distant from $P$. falciparum, the MSP-4 homologue was located using the adenylosuccinate lyase (ASL) gene as a probe and successfully carrying out a cross-species hybridization (Black et al., 2002). PvMSP-4 gene also includes a two exon structure and encodes proteins with potential signal and GPI anchor sequences and a single EGF-like domain near the carboxyl-terminus. Rabbit anti sera raised against purified recombinant protein showed that each of the antisera reacted to a $62 \mathrm{kDa}$ protein in parasite lysates. In the same study indirect immunofluorescence assays (IFA) localized PvMSP-4 over the entire surface of $P$. vivax merozoites (Black et al., 2002).

Immunoepidemiology investigates the influence of population immunity on epidemiological patterns (Zetkin et al., 1992). Monitoring of immune status at intervals allows the planning and evaluation of intervention programmes, discovery of antigenic variation resistant genotypes associated with parasite protection from host immunity. This in turn helps to identify antigens that can give rise to highest response when introduced as vaccines. Further, a detailed understanding of naturally acquired immunity in individuals and populations is important for planning vaccine development (Hellriegel, 2001).

We report a cross sectional study examining for the first time the naturally acquired antibody responses to $P$. vivax MSP-4 by ELISA using baculovirus expressed recombinant protein, in test populations living in two malaria endemic regions (Anuradhapura and Kataragama) and a nonendemic region (Colombo) in Sri Lanka. Using this same battery of sera, we have previously investigated the antibody responses against the Cterminal processing products of $P$. vivax MSP-1 represented by two recombinant proteins, $\mathrm{p} 42$ and p19 (Wickramarachchi et al., 2007). Attempts were also made to draw comparisons among the nature of the antibody responses to MSP-4, p42 and $\mathrm{p} 19$ in the same population of acute vivax malaria patients.

\section{MATERIALS AND METHODS}

\section{Sample Collection}

Following ethical approval by the ethics review committee of the University of Colombo, Sri Lanka, (EC/04/103), blood samples were collected with informed consent from $P$. vivax infected patients (> 15 yrs. Of age) presenting during 1998-2001 from two endemic areas, the General Hospital, Anuradhapura $\left(8^{\circ} 22^{\prime} \mathrm{N}\right.$, $\left.80^{\circ} 20^{\prime} \mathrm{E} ; \mathrm{N}=68\right)$, Malaria Research station, Kataragama ( $6^{\circ} 25^{\prime} \mathrm{N}, 81^{\circ} 20^{\prime} \mathrm{E}$; $\left.\mathrm{N}=87\right)$, and a nonendemic area Colombo $\left(7^{\circ} 55^{\prime} \mathrm{N}, 79^{\circ} 50^{\prime} \mathrm{E}\right.$; $\mathrm{N}=69$ ) (Wickramarachchi et al., 2006). The majority of patients from Colombo, which is malaria free (Briet et al., 2003, Fonseka et al., 1987), were adults returning from visits to regions with $P$. vivax transmission. Blood samples from healthy individuals with no history of malaria were used as normal controls $(\mathrm{N}=30)$. Blood was drawn 
under aseptic conditions by venipuncture and serum samples were stored at $-20^{\circ} \mathrm{C}$ until further use.

As most malaria infections are symptomatic in patients from Sri Lanka (Mendis et al., 1990, Gunewardena et al., 1994) and malaria episodes are routinely confirmed by microscopy prior to treatment, the self-reported number of past clinical episodes ( $P$. vivax and $P$. falciparum) was used to classify these patients in to four categories based on number of previous exposure to malaria: i) 0 infections, ii) 1-2 infections, iii) 3-5 infections, iv) $>5$ infections.

\section{Recombinant PvMSP-4}

A synthetic gene coding for the entire 230 amino acid PvMSP4 antigen was constructed was cloned into the pVL1393 baculovirus transfer plasmid (Bam H1-EcoR1) and used to create recombinant baculovirus as described (Longacre et al, 1994). PvMSP4 was recovered in culture supernatants of High five insect cells infected with the recombinant baculovirus and purified to homogeneity using metalloaffinity chromatography. Antibody recognition of baculovirus PvMSP4 in sera from 17 randomly chosen $P$. vivax infected individuals, tended to be reduction sensitive (12/17), suggesting that the recombinant antigen carried a correctly folded EGF-domain (having three disulfide bridges).

\section{Determination of total anti-PvMSP-4 antibodies by indirect ELISA}

The total anti-MSP-4 antibody content (IgM+IgG) in the sera was quantified by an indirect microplate assay ELISA as previously described (Wickramarachchi et al., 2006), using recombinant protein PvMSP-4 representing conformation of the native protein, as antigen. Each serum sample was assayed against the protein in duplicate, with 30 normal human sera as controls.

Checkerboard titrations determined the optimal dilutions of reagents used in this ELISA, i.e., $1.2 \mu \mathrm{g} / \mathrm{ml}$ antigen, serum samples at 1:100 dilution. The cut-off value was calculated as the mean OD value of the normal controls plus 2 standard deviations. The mean OD value at $415 \mathrm{~nm}$ obtained at serum dilution of 1:100 was considered a measure of the magnitude of the anti-MSP-4 antibody responses.

\section{Statistical analysis}

Statistical analyses of data were performed using SPSS 13 for windows (SPSS Inc., USA) and
Epi Info 6 (version 6.04b to c upgrade; CDC, USA and WHO, Switzerland) computer programmes.

Proportions of responders for independent samples were compared using the Chi-squared test, and paired comparisons were made with the McNemar test. Comparisons of antibody magnitudes of independent samples were performed using one-way ANOVA and MannWhitney U test, and as appropriate Kruskal-Wallis $\mathrm{H}$ test was used for dependent samples. Associations between antibody responses and prevalence with host factors were derived using Spearman correlation coefficient and Chi-squared for linear trend, respectively. The significance level was set at $\mathrm{P}<0.05$.

\section{RESULTS}

\section{Total (IgM + IgG) antibody responses to PvMSP-4}

During acute infection, prevalence (responding percentages of antibody-positive sera) to PvMSP4 was $80 \%, 62 \%$ and $62 \%$ from Colombo, Anuradhapura and Kataragama, respectively (Table. 1). Significantly higher prevalence (Chisquared test, $\mathrm{P}<0.05$ ) and antibody magnitude (Kruskal-Wallis test, $\mathrm{P}<0.05$ ) was recorded in non-endemic Colombo compared with both the endemic areas. There was no significant difference ( $\mathrm{P}>0.05)$ either in prevalence or in antibody magnitude between individuals who were previously not exposed (PNE) to malaria in Colombo and their previously exposed counterparts (PE) from the same area. A significantly higher antibody magnitude (KruskalWallis test, $\mathrm{P}<0.05$ ) but no significant difference in prevalence $(\mathrm{P}>0.05)$ was evident in the $\mathrm{PNE}$ group than of the two endemic groups.

\section{Associations between total antibody responses and host factors}

In all test populations, antibody magnitudes were evaluated with the patient's age, previous exposures to malaria, and parasitaemia.

There was no correlation between total PvMSP-4 antibody magnitude in any of the three test areas, either with age of the patient or with their days of symptoms (Spearman Correlation coefficient, $\mathrm{P}>0.05$ ). No significant association was found between parasitaemia and the antibody magnitude in Colombo and Anuradhapura (Spearman Correlation coefficient, $\mathrm{P}>0.05$ ), whereas in Kataragama there was a positive correlation between parasitaemia and the antibody 
magnitude (Spearman Correlation coefficient, $\mathrm{P}<0.05$ ).

There was no significant association between antibody magnitude and the number of previous exposure to malaria in the three study areas (Spearman Correlation coefficient, $\mathrm{P}>0.05$ ), however, a positive trend was evident in Kataragama while a negative trend was evident from Colombo to responding proportions and previous exposure to malaria infections, but this trend was not significant (Chi squared for linear trend, $\mathrm{P}>0.05$ ) (Fig.1).

Comparison of antibody responses to $P$. vivax asexual stage vaccine candidate antigens (MSP4, MSP-1p42 and p19)

Analysis was carried out using the same battery of sera to compare the naturally acquired antibody response of each $P$. vivax infected patient with respect to different asexual stage vaccine candidates PvMSP-1p42, PvMSP-1p19 (Wickramarachchi et al., 2007) and PvMSP-4 during the same episode of malaria. For this analysis antibody response of 45, 84 and 30 individuals, respectively, from malaria endemic Anuradhapura, Kataragama and non-endemic Colombo were included.

The percentage of responders for antibodies against each antigen in different test areas revealed that, $66 \%, 47 \%$ and $44 \%$ of the test populations from Colombo, Anuradhapura and Kataragama, respectively, responded to all three antigens. However, more than $80 \%$ from each test area responded to at least one of the three antigens tested. The antibody prevalence in all test areas for p42 was significantly higher (McNemar Test $\mathrm{P}<0.05$ ) than that for the other two antigens (Fig. 2). Importantly, $7 \%, 11 \%$ and $4 \%$ of individuals respectively, from Colombo, Anuradhapura, and Kataragama, preferentially recognized PvMSP-4 (Chi-squared test, $\mathrm{P}<0.05$ ). No significant differences were apparent $(\mathrm{P}>0.05)$ among responding proportions to PvMSP-4, p42, and p19 among the three study areas. Of the PNE individuals from Colombo, 45\% responded to all antigens, while $9 \%$ did not respond to any. Individuals with previous exposure from Colombo showed a significantly higher antibody prevalence (Chi-squared test, $\mathrm{P}<0.05$ ) to all three antigens compared with both endemic areas. Further, all individuals from Colombo with previous history of malaria responded to at least one of the antigens tested.

Responders to paired combinations of PvMSP1 and PvMSP-4 were analyzed (Figure 3), and the percentage of responders to MSP-1p42 + p19 in paired combination showed higher prevalence compared to paired combination of MSP-1 with MSP-4 (Chi-squared test, $\mathrm{P}<0.05$ ).

Table 1. Prevalence and antibody magnitude of total (IgM+IgG) anti PvMSP-4 antibodies of acute Plasmodium vivax patients from the three study areas.

\begin{tabular}{lccl}
\hline \multicolumn{4}{c}{ PvMSP-4 } \\
\hline Area & No. of Samples & Prevalence \% & Antibody level $^{\mathrm{a}}$ \\
\hline Endemic & & & \\
Anuradhapura & 68 & 62 & $0.375( \pm 0.058)$ \\
Kataragama & 87 & 62 & $0.323( \pm 0.036)$ \\
& & & \\
Non-endemic & & & \\
Colombo-total & 69 & 80 & $0.561( \pm 0.068)$ \\
PNEb & 25 & 76 & $0.6( \pm 0.112)$ \\
PEc & 37 & 78 & $0.581( \pm 0.1)$ \\
\hline
\end{tabular}

\footnotetext{
a The mean OD $405 \mathrm{~nm}$ value obtained at a serum dilution of 1:100 was considered as a measure of the level of the anti PvMSP-4 total antibody response of each individual. The cut-off value was the mean $\mathrm{OD}_{405 \mathrm{~nm}}$ value plus 2 SD of the healthy individuals from Colombo, with no past history of malaria. The antibody level of each test area is expressed as means \pm SEM

${ }^{\mathrm{b}}$ Previously not exposed to malaria

${ }^{\mathrm{c}}$ Previously exposed to malaria
} 

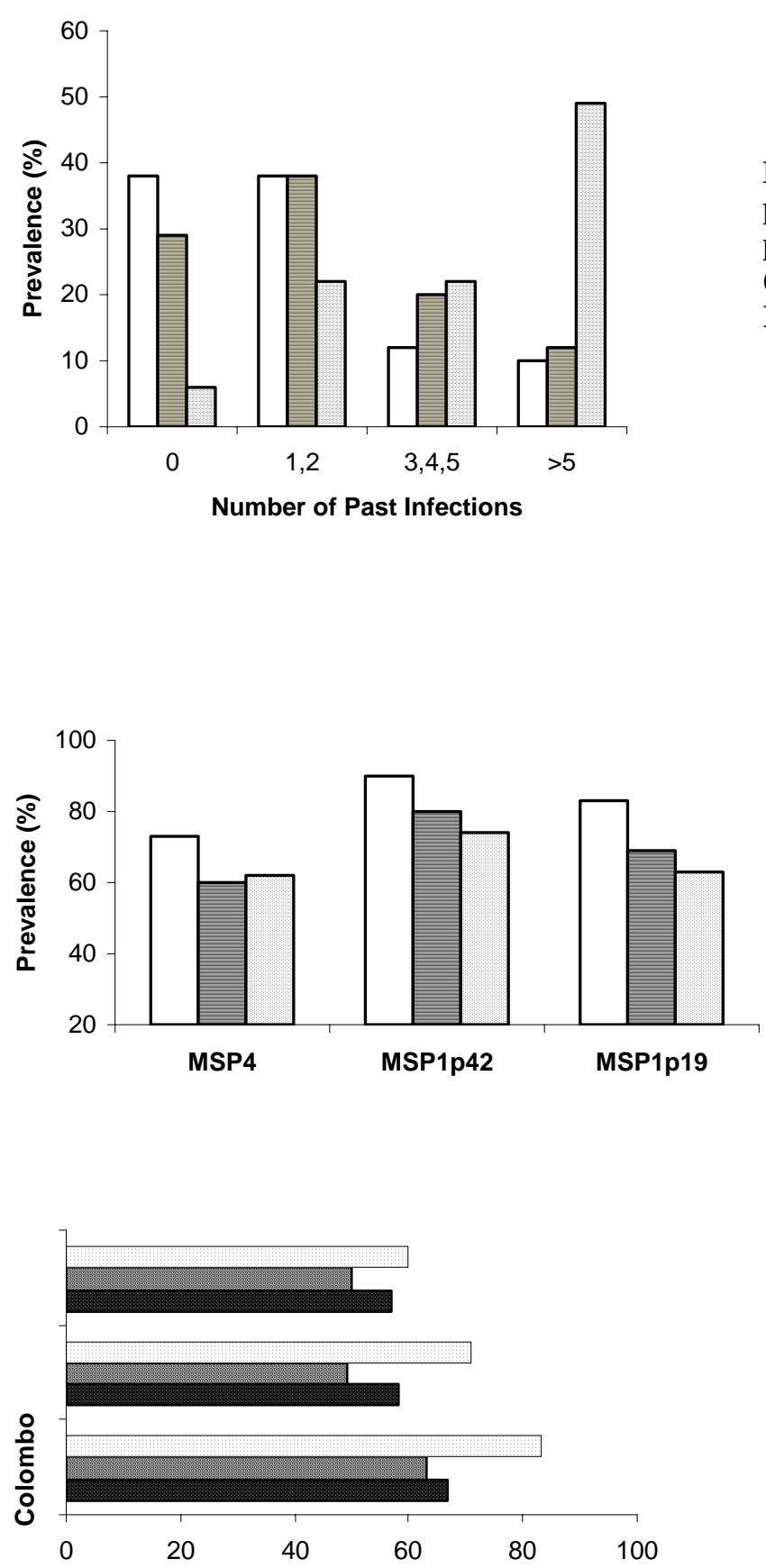

Figure 1. Anti PvMSP-4 antibody prevalence against different numbers of previous malaria infections in Colombo ( $\square$ ), Anuradhapura (回) and Kataragama ( $\square$ ).
Figure 2. Antibody prevalence to PvMSP-4, PvMSP-1p42 and, PvMSP-1p19 in different study areas. Colombo ( $\square$ ), Anuradhapura (国) and Kataragama ( $\square$ )

\section{DISCUSSION}

This is the first study to examine the natural antibody responses to PvMSP-4 in endemic and non-endemic residents of Sri Lanka suffering acute vivax malaria infections. The present study, aimed to identify association(s) between antiPvMSP-4 antibody prevalence and magnitude in combination with host factors (age, parasite density, number of previous infections of malaria) in order to establish contributory factors giving rise to "protection" in vivo of $P$. vivax infected individuals of endemic areas of Sri Lanka. Importantly, compared to PfMSP-4 antigen (Marshall et al., 1997; Wang et al., 1999 \& 2001), there is no published immuno-epidemiological 
data on antibody responses to PvMSP-4, especially using an antigen likely to have the native conformation.

It is widely believed that a large proportion of the naturally acquired immunity to the asexual blood stage parasites is antibody based (Anders et al., 1993). Therefore, this study focused on the antibody responses to PvMSP-4, although cellmediated immune responses may also exist and play a role in immune protection (Wang et al., 2001). It was observed that anti-MSP-4 antibodies are present at high levels in individuals living in malaria endemic as well as non-endemic areas of the island, suggesting that PvMSP-4 is a well recognized asexual stage parasite antigen. The lack of antibody response to PvMSP-4 in some of the individuals in these test areas is unlikely to be due to lack of exposure since all residents were exposed to the parasite repeatedly.

Antibody prevalence against PvMSP-4 did not show any significant difference (Chi squared Test, $\mathrm{P}>0.05$ ) among the three study groups. Anuradhapura and Kataragama are typical $P$. vivax malaria endemic areas in Sri Lanka (Gunawardena et al., 1994). Residents living in non-endemic Colombo, which is free of malaria transmission, acquire malaria through visiting endemic areas, and the majority who had suffered multiple attacks of malaria was frequent visitors to malaria endemic areas due to their occupations (Wickramarachchi et al., 2006). Therefore, comparable antibody prevalence between individuals from endemic and non-endemic areas was unexpected as our hypothesis was that total antibody prevalence would be lower among individuals from non-endemic Colombo. However, this study showed otherwise, with higher prevalence $(80 \%)$ in Colombo compared with the two endemic areas (Anuradhapura 62\%, and Kataragama 62\%). In addition, significantly higher antibody prevalence among individuals from Colombo with previous exposure (PE) to malaria than those individuals lacking previous exposure was observed. This indicates that, though previous exposure to malaria could be a contributory factor for the presence of antibodies against PvMSP-4, this antigen was immunogenic even in certain individuals with limited antigenic exposure (Wickramarachchi, 2005). Higher levels of antibody (Kruskal-Wallis test, $\mathrm{P}<0.05$ ), but no significant difference in prevalence, was evident between the previously not exposed individuals (PNE) from Colombo and the two endemic groups. A plausible explanation for the low response in the endemic areas may be due to genetic restriction established in these populations, which limits the antibody production (Richie and Saul, 2002). Previous immuno-epidemiological studies (Egan et al., 1995; Shi et al., 1996; Taylor et al., 1998; Wang et al., 2001) have indicated that the lack of $100 \%$ antibody prevalence to malaria antigens against highly exposed populations to malaria probably reflects genetic control of host antibody responses. Nevertheless, a study of $P$. falciparum MSP-4 reported a higher antibody prevalence among individuals living in an endemic region of Vietnam (Escherichia coli expressed recombinant MSP-4 (EcMSP-4) - 82\% and Saccharomyces cerevisiae expressed recombinant MSP-4 (ScMSP-4) - 94\%) (Wang et al., 2001). On the contrary, it has also been shown that imprinting of a positive or a non-response to a specific malarial antigen is independent of the host genotype (Riley et al, 1994).

Although there was no serological data for children in this study (due to ethical and pragmatic reasons), the very low inoculation rates (Mendis et al., 1990) and heterogeneous exposure among different age groups in Sri Lanka (Mendis et al., 1992) make it unlikely that such an age correlation exists in these settings. Low transmission rates in Sri Lanka (Mendis et al., 1990) can also explain the lack of association between antibody levels specific for MSP-4 clinical episodes and parasitemia from the three test areas, as acquisition of protective anti parasite immunity may need a high level of endemicity (Wickramarachchi, 2005). A positive correlation for parasitemia with antibody magnitude was observed among residents of Kataragama (Spearman Correlation coefficient, $\mathrm{P}>0.05$ ). This observation may merely be an indicator of the parasite load present in each area. However, no significant correlation was apparent in Colombo and Anuradhapura. A prospective study on selected endemic sub populations is required to reach more definitive conclusions on antibody responses and manifestation of parasite loads as certain protective trends prevailing within local endemic populations may not be distinguished in a broad cross sectional study.

No significant association was found between antibody responses and previous exposure to malaria in the three study areas. Nevertheless, a positive trend was evident in Kataragama (Chi square for linear trend, $\mathrm{P}>0.05$ ) suggesting antibody maturation (Abbas et al., 2000) and/or "immunosupression / immunodepression" (Pachner, 1997). Furthermore, this positive trend is indicative of immunological memory. 
The response of PvMSP-4 in combination with PvMSP-1 (p42 and p19) was analyzed using the same battery of sera to evaluate the feasibility of a multivalent vaccine of these antigens. The antibody prevalence against each antigen presented illustrates that the antibody responsiveness to PvMSP-1p42 was significantly higher in all three test areas compared with the other two vaccine candidates tested (McNemar test, $\mathrm{P}<0.05)$. Therefore, PvMSP-1p42 as a potential vaccine candidate might be better able to boost vaccine induced immunity during natural malaria infections in individuals than the other two antigens tested among populations residing both in endemic and non-endemic areas of Sri Lanka. Previous reports had confirmed this conclusion (Egan et al., 1995, Wickramarachchi et al., 2007). However, PvMSP1p42 carries a highly polymorphic region that is highly immunogenic, perhaps limiting the usefulness of this antigen as vaccine candidate (A. Manamperi, personal communication). Comparison of antibody prevalence of PvMSP-1p19 and PvMSP-4 indicate that both these vaccine candidates are comparatively immunogenic in individuals from endemic as well as non-endemic areas though less immunogenic than PvMSP-1p42. All individuals from Colombo with a previous history of malaria responded to at least one of the antigens tested. However, why such high antibody prevalence occurred among PE individuals from Colombo remains to be elucidated.

The percentage of responders to PvMSP-1p42 and p19 in paired combination showed higher prevalence compared with paired combination of PvMSP-1 with PvMSP-4 (Chi-squared test, $<0.05$ ), illustrating that PvMSP-1p42 + p19 as a potential vaccine construct would be able to boost vaccine induced immunity during natural malaria infections.

Serological data indicate that intrinsic properties of the protein antigen itself play a major role in determining the subclass profile of the antibody response, which has important implications for the rational design of vaccine delivery. Therefore, isotype analyses as well as prospective immunoepidemiological studies are warranted to gain in depth knowledge of the antibody response to PvMSP-4. The present study provides essential base line information required for a future clinical trial for a multi-component vaccine based on MSP-1 and MSP-4 of P. vivax blood stage vaccine candidates.

\section{ACKNOWLEDGEMENTS}

Financial assistance from the National Science Foundation, Sri Lanka (NSF/RG/2005/HS/06) and the Institute of Biochemistry Molecular Biology and Biotechnology, University of Colombo is acknowledged. The assistance rendered by the staff of the Department of Zoology, Faculty of Science, University of Colombo and the Institute of Biochemistry Molecular Biology and Biotechnology, University of Colombo is deeply appreciated. We are grateful to Dr Thilan Wickramarachchi for collecting the serum samples, and to Dr Shiroma Handunnetti, former Head of the Malaria Research Unit, Faculty of Medicine, University of Colombo. The help and support given by Prasad $\mathrm{H}$ Premaratne of our group is deeply appreciated.

\section{REFERENCES}

Abbas, A.K., Lichtman, A.H. and Pober, J.S. (2000). Cellular and Molecular Immunology, $4^{\text {th }}$ edition, W. B. Saunders Company, New York. Pp.1999-207.

Anders, R.F. and Saul, A.J. (1993). Candidate antigens for an asexual blood stage vaccine against falciparum malaria. In molecular immunological considerations in malaria vaccine development. Good, M.F., and Saul, A.J. (Eds). Boca Raton: CRC Press. Pp. 169-208.

Anders, R.F. and Saul, A.J. (2000). Malaria vaccines. Parasitology Today 16: 444-447.

Annual Health Bulletin Sri Lanka. Ministry of Health, 2002. Pp. 50-52.

Barnwell J.W., Galinski, M.R., De Simons S.G., Perler, F. and Ingravallo, P. (1999). Plasmodium vivax, $P$. cynomolgi, and $P$. knowlesi: identification of homologue proteins associated with the surface of merozoites. Experimental Parasitology 91: 238-249.

Black, C. G., Barnwell, J.W., Huber, C. S., Galinski, M. R. and Coppel, R. L. (2002). The Plasmodium vivax homologues of merozoite surface proteins 4 and 5 from Plasmodium falciparum are expressed at different locations in the merozoite. Molecular and Biochemical Parasitology 120:215-224.

Briët, O. J. T., Gunawardena, D. M., van der Hoek, W. and Amerasinghe, P. M. (2003). Sri 
Lanka malaria maps. Malaria Journal; 2: 22. [Online.]http:// www.malariajournal.com/ content/ $2 / 1 / 22$.

Egan, A. F., Chappel, J. A. and Deleron, T. C. (1995). Serum antibody from malaria-exposed people recognized conserved epitopes formed by the two epidermal growth factor motifs of MSP-1 (19) the carboxyl terminal fragment of the major merozoite surface protein of Plasmodium falciparum. Infection and Immunity 63(2): 456466.

Fonseka, J., Mendis, K.N. (1987). A metropolitan hospital in a non-endemic area provides a sampling pool for epidemiological studies on vivax malaria in Sri Lanka. Transaction of the Royal Society of Tropical Medicine and Hygiene 81:360-364.

Gunawardena, D. M., Carter, R. and Mendis, K. N. (1994). Patterns of acquired anti-malarial immunity in Sri Lanka. Mem Inst Oswaldo Cruz 89: $61-63$

Hellriegel, B. (2001). Immunoepidemiologybridging the gap between immunology and epidemiology. Trends in Parasitology 17: $102-$ 106.

Hoffman, S., Coppel, R. L. and Chulay, J. (1991). Vaccines. In Oaks, S.C., Mitchell, V. S., Pearson, G. W. and Carpenter, C. C. J (Eds), Malaria: Obstacles and opportunities. National Academy Press, Washington, D.C. Pp.16-210.

Hoffman, S. L. K., Subramanian, G. M., Collins, F. H. and Venter, J. C. (2002). Plasmodium, human and Anopheles genomics and malaria. Nature 415:702-709.

Konradsen, F., Amarasinghe, F.P., Van der Hoek, W. and Amaresinge, P. H. (2000). Malaria in Sri Lanka, Current knowledge on transmission and control. Colombo, Sri Lanka; International Water Management Institute. Pp.21-36.

Longacre, S., Mendis, K.N. and David., P.H. (1994). Plasmodium vivax merozoite surface protein 1 C-terminal recombinant proteins in baculovirus. Molecular Biochemistry Parasitology 64:191-205.

Marshall, V. M., Silva, A., Foley, M., Cranmer, S., Wang, L., McColl, D. J., Kemp, D. and Coppel, R. L. (1997). A second Merozoite Protein (MSP4) of Plasmodium falciparum that contains an epidermal growth factor -like domain. Infection and Immunity 65: 4460-4467.

Mendis, M., Sina, J. S., Marchesini, M. and Carter, R. (2001). The neglected burden of Plasmodium vivax malaria. American Journal of Tropical Medicine and Hygiene 64: 97-105.

Mendis, C., Giudice G.D., Gamage-Mendis, A.C., Tougne, C., Pessi, A., Weerasinghe, S., Carter, R. and Mendis, K.N. (1992). Anti-circumsporozoite protein antibodies measure age related exposure to malaria in Kataragama, Sri Lanka. Parasite Immunology 14:75-86.

Mendis, C., Giudice, G. D., Gamage-Mendis, A. C., Tougue, C., Pessi, A., Weerasinghe, S., Carter, R. and Mendis, K. N. (1990). Anticircumsopozoite protein antibodies measure agerelated exposure to malaria in Kataragama, Sri Lanka. Parasite Immunology 14: 75-86.

Pachner, A.R. (1997). Immunosuppresive therapy: Targeting the autoantigen in neurologic disease. Advance. Neuroimmunol. 4: 3-8.

Rajendram, S. and Jayewickreme, S. H. (1951). Malaria in Ceylon. Indian Journal of Malariology 5: $1-2$.

Richie, T. L. and Saul, A. (2002). Progress and challenges for malaria vaccines. Nature 415: 694700 .

Riley E.M., Bennett S., Jepson A., Hassan-King M., Whittle H., Olerup O. and Carter R. 1994. Human antibody responses to Pfs 230, a sexual stage-specific surface antigen of Plasmodium falciparum: non-responsiveness is a stable phenotype but does not appear to be genetically regulated. Parasite Immunology16:55-62.

Shi, Y.P., Sayed, U., Qari, S.H., Roberts, J.M, Udayakumar, V, Oloo, A.J., Hawley, W.A., Kaslow, D.C., Nehlen, B.L. and Lal, A.A. (1996). Natural immune response to the C-terminal 19kilodalton Domain of Plasmodium falciparum Merozoite Surface Protein 1. Infection and Immunity 64: :2716-2723.

Taylor, R. R., Allen, S. J., Greenwood, B. M. and Riley, E. M. (1998). IgG3 antibodies to Plasmodium falciparum Merozoite Surface Protein 2 (MSP2): increasing prevalence with age and association with clinical immunity to malaria. The American Journal of Tropical Medicine and Hygiene 58: 406-413. 
Wang, L., Kedzierski, L., Wesselingh, S. L. and Coppel, R. L. (2003). Oral immunization with a recombinant malaria protein induces conformational antibodies and protects mice against lethal malaria. Infection and Immunity 71(5): 2356-2364.

Wang, L., Richie, T. L., Stowers, A., Nhan, D. H. and Coppel, R. L. (2001). Naturally acquired antibody responses to Plasmodium falciparum Merozoite Protein 4 in a population living in an area of endemicity in Vietnam. Infection and Immunity 69: 4390-4397.

Wang, L., Black, C. D., Marshall, V. K. and Coppel, R. L. (1999). Structural and antigenic properties of Merozoite Surface Protein 4 of Plasmodium falciparum. Infection and Immunity 67: 2193-2199.

Waters, A. (2006). Malaria: New vaccines for old? Cell 124: 689-693.

Wickramarachchi, T., Illeperuma, R.J., Perera, L., Bandara, S., Holm, I., Longacre, S., Handunnetti, S.M. and Udagama-Randeniya, P.V. (2007). Comparison of naturally acquired antibody responses against the C-terminal processing products of Plasmodium vivax Merozoite Surface
Protein-1 under low transmission and unstable malaria conditions in Sri Lanka. International Journal for Parasitology 37:199-208.

Wickramarachchi, T., Premaratne, P.H., Perera, K. L. R. L., Bandara, S., Kocken, C. H. M., Thomas, A. W., Handunnetti, S. M. and UdagamaRandeniya, P. V. (2006). Natural human antibody responses to Plasmodium vivax Apical Membrane Antigen 1 under low transmission and unstable malaria conditions in Sri Lanka. Infection and Immunity 74 (1): 798-801.

Wickramarachchi, W. T. A. (2005). Immuno and molecular epidemiology of Plasmodium vivax blood stage antigens under low transmission and unstable malaria conditions of Sri Lanka (PhD Dissertation); University of Colombo, Colombo, Sri Lanka.

Wickremasinghe, R.A. (2002). Burden of P. vivax malaria in Sri Lanka. Vivax malaria research: 2002 and beyond, Siam city hotel, Bangkok, Thailand. Pp.2.

Zetkin, M. and Schaldach, H. (1992). Woerterbuch der Medizin. 5th Ed. Berlin: Ullstein Mosby. 